Please do not remove this page

RMIT

UNIVERSITY

\title{
Fractal dimension of the retinal vasculature and risk of stroke: A nested case-control study
}

Kawasaki, Ryo; Che Azemin, Mohd Zulfaezal; Kumar, Dinesh; Tan, Audrey; Liew, G; Wong, W; Mitchell, P https://researchrepository.rmit.edu.au/esploro/outputs/9921857612101341/filesAndLinks?institution=61RMIT_INST\&index=null

Kawasaki, R., Che Azemin, M. Z., Kumar, D., Tan, A., Liew, G., Wong, W., Mitchell, P., \& Wang, T. (2011). Fractal dimension of the retinal vasculature and risk of stroke: A nested case-control study. Neurology, 76(20), 1766-1767. https://doi.org/10.1212/WNL.0b013e31821a7d7d

Document Version: Submitted Version

Published Version: https://doi.org/10.1212/WNL.0b013e31821a7d7d

Repository homepage: https://researchrepository.rmit.edu.au (C) 2011

Downloaded On 2023/04/27 00:06:38 +1000 
NEUROLOGY MS ID\# : NEUROLOGY/2010/354571

\section{Fractal Dimension of the Retinal Vasculature and Risk of Stroke:}

A Nested Case-control study.

Ryo Kawasaki, PhD, ${ }^{1,2}$ Mohd Zulfaezal Che Azemin, MSBE, ${ }^{3}$ Dinesh Kant Kumar, $\mathrm{PhD},{ }^{3}$ Ava Grace Tan, MPH, ${ }^{4}$ Gerald Liew, PhD, ${ }^{4}$ Tien Yin Wong, $\mathrm{PhD},{ }^{1,5}$ Paul Mitchell, $\mathrm{PhD},{ }^{4}$ Jie Jin Wang, $\mathrm{PhD}^{1,4}$

1. Centre for Eye Research Australia, University of Melbourne, Victoria, Australia

2. Royal Victorian Eye and Ear Hospital, Victoria, Australia

3. School of Electrical and Computer Engineering, RMIT University, Victoria, Australia

4. Centre for Vision Research, University of Sydney, NSW, Australia

5. Singapore Eye Research Institute, Singapore National Eye Centre, Singapore

Correspondence to: Jie Jin Wang, MMed (Clin Epi), MAppStat, $\mathrm{PhD}$

Centre for Eye Research Australia, University of Melbourne

32 Gisborne Street, Victoria 3002 Australia

Tel: +6139929 8352, Fax: +61396623859

Email: jiejw@unimelb.edu.au

Conflict of Interest/Disclosure: None

Funding:

Word counts: 924/750; References: 7/7; Table: 1/1; Supplemental on-line Tables/Figures: 2/1 
Authors' disclosure:

Dr Kawasaki is funded by NHMRC grant \#590212, Grant-in-Aid for scientific research, Japan Society for the Promotion of Science Projects grants \#22591960, \#22659135, and Foundation for Total Health Promotion Project grant, Japan.

Mr. Azemin reports no disclosures.

Dr. Kumar serves as an associate editor of IEEE Transactions of Neural Systems and

Rehabilitation Engineering and Journal of medical and Biological Engineering, is funded by ARC Linkage grant, and serves as consultant for the ATOS Ltd, Intelligent Systems Pty Ltd, and Aquarian Industries Pty Ltd.

Mis. Tan reports no disclosures.

Dr. Liew is funded by NHMRC Project grant \#475604 and Hear Foundation.

Dr. Wong serves on the scientific advisory board for Novartis, Pfizer, Allergan and Bayer, and received research and travel support from the these companies.

Dr. Mitchell serves as a section editor for the British Journal of Ophthalmology, is funded by NHMRC Project grants \#512530, \#475604, \#571012, 571013, NHMRC Centres for Clinical Research Excellence \#529923, NHMRC Partnership Project grants \#571449, \#569944, Mason Foundation, Dairy Australia and Heart Foundation.

Dr. Wang is funded by NHMRC Senior Fellowship \#632909, NHMRC Project grants \#512423, \#512530,\#475604, \#590204, NHMRC Development grant \#475617, NHMRC Centres for Clinical Research Excellence \#529923, NHMRC Partnership Project grant \#571449, Diabetes Australia Research Trust, the Jack Brockhoff Foundation Grant, Juvenile diabetes Research Foundation International, Westmead Charitable Trust, Heart Foundation, and American Health Assistance Foundation. 


\section{INTRODUCTION}

Recent studies show associations between retinal vascular changes and either small infarcts detected on brain imaging, or with clinical stroke. ${ }^{1}$ The retinal vascular fractal dimension has been used as a global measure of the geometric pattern of the vasculature ${ }^{2,3}$ potentially representing the complexity of the branching pattern of vessels in the body, including the cerebral microvasculature. A loss of complexity of the cerebral microvasculature may lead to limited collateral formation at cerebral infarction sites. ${ }^{4}$ To date, studies have reported cross-sectional associations between retinal fractal dimension and lacunar stroke. ${ }^{4,5}$ However, no prospective data are available about the relation of retinal fractal dimension and the incidence of stroke.

A limitation of retinal vessel measurement is measurement noise in segmentation; we therefore developed a method to use the Fourier-transformed images to assess spectrum fractal dimension (SFD) of the retinal vasculature ${ }^{6,7}$ With this new measurement, we expect to detect vasculature changes that may signal a risk of future stroke. In this study we examined the association between baseline SFD and stroke incidence using a case-control sample nested in the Blue Mountains Eye Study (BMES) cohort.

\section{METHODS}

The BMES is a population-based cohort study of an urban Australian population aged 49 years or older $(n=3,654)$, representing $82.4 \%$ of eligible population in a defined two-postcode area of the Blue Mountains region, Australia. ${ }^{1}$ Written informed consent was obtained from all participants at enrollment. ${ }^{1}$

Stroke cases were defined among participants who had no history of stroke at baseline (1992-94) but who developed incident stroke over the subsequent 5 years (1997-99), or who had died from stroke or stroke-related causes in the period up to December 31,2005. The definitions used to identify stroke events and mortality are shown in Table e-1. We identified 130 cases with either stroke event or stroke-related mortality, however 26 persons were excluded due to missing 
photographs or clinical information on hypertension or diabetes. As a result, we included 21 stroke events and 86 stroke-related deaths, including 3 persons overlapped in both, which gave a total of 104 cases. Two controls per case were selected from the remaining participants matched for age, gender, diabetes and hypertension status. Of these, $97.1 \%(101 / 104)$ cases and $88.5 \%$ $(184 / 208)$ controls had digitized, baseline retinal photographs of sufficient quality to enable SFD assessment. Images centered at the optic disc were re-sampled to 778 x 519 pixels for efficient image processing. An area centered on the optic disc covering 2.5 disc radii was cropped and enhanced using the Gabor-wavelet transform ${ }^{6,7}$ (Figure e-1). The SFD, a mono-fractal of retinal vascular image spectrum, represents a slope of linear association between the natural log-scaled image intensity ("magnitude") and the natural log-scaled pixel density frequency distribution ("frequency"). ${ }^{6,7}$ This system is fully automated. To assess the reproducibility of image cropping, we performed a Monte Carlo type simulation for image cropping; the repeated estimates of SFD were highly replicated on 100 simulated grading with a correlation of 0.93 . $^{7}$

\section{Statistical analysis}

We used SFD measures from the left eye, given the high correlation between two eyes $(r=0.63)$. We constructed conditional logistic regression models to determine odds ratio (OR) and 95\% confidence intervals (CI) for each SD decrease in SFD or for lower quartiles: unadjusted (Model 1) or adjusted for stroke risk factors (Model 2: body mass index, smoking, total cholesterol, highdensity-lipoprotein cholesterol, and triglycerides; Model 3: plus systolic blood pressure and glucose level; and Mode 4: further plus age). Stepwise selection method was applied in Model 4 to identify significant risk factors for incident stroke. Stata 11.1 (StataCorp, College station, TX) was used.

\section{RESULTS}

Overall the mean age was 73.8 (standard deviation [SD] 8.2) years, $58 \%$ were female, $49.5 \%$ were hypertensive and $4.6 \%$ had diabetes. There were no significant differences in the matched 
characteristics or other stroke risk factors between cases and controls (Table). The mean SFD was $1.509(\mathrm{SD} 0.026)$.

Cases had significantly smaller SFD (1.504) compared to controls (1.511; $\mathrm{p}=0.044)$. Each SD decrease in baseline FFD was associated with $40 \%$ greater risk of stroke (OR 1.39, 95\%CI 1.061.83). This remained statistically significant after adjusting for stroke risk factors (Model 4: OR $1.36,95 \%$ CI $1.00-1.84)$. Persons in the smallest quartile of SFD $(<1.494)$ were twice more likely to have a subsequent stroke than those with the largest quartile ( $>1.527)$ (stroke incidence $42.3 \%$ vs. 27.8\%; OR 2.30, 95\% CI 1.06-4.97) (Table e-2). This association remained significant after adjusting for body mass index, smoking, total cholesterol, HDL cholesterol, and triglycerides (Model 2: OR 2.42,95\% CI 1.04-5.62), or further adjusting for systolic blood pressure and glucose (Model 3: OR 2.43, 95\% CI 1.04-5.68). However, it became attenuated after further adjusting for age (Model 4: OR 2.13, 95\% CI 0.90-5.04). Using the stepwise backward selection method, the incremental (per SD decrease) in the FFD was the only significant factor remained.

\section{DISCUSSION}

We found that low SFD of retinal vasculature was associated with 2-fold risk of incident stroke compared to persons with high SFD, independent of stroke risk factors. Previous cross-sectional studies found inconsistent findings between retinal vascular fractal dimension and lacunar stroke ${ }^{4}$,

${ }^{5}$. We provide the first longitudinal data in this regard, although limitations present, including potential selection bias from excluding cases with poor quality images, and the lack of stroke subtype information.

While our study supports the concept that structural retinal microvascular changes may represent a subclinical biomarker of stroke, what SFD changes indicate in the context of stroke pathogenesis remains unclear. We speculate that reduced SFD indicates reduced complexity in branching pattern of the microvasculature, leading to reduction in perfusion and limited ability to form collaterals, which could increases vulnerability to cerebral hypoxia. ${ }^{6}$ 


\section{REFERENCES}

1. Mitchell P, Wang JJ, Wong TY, Smith W, Klein R, Leeder SR. Retinal microvascular signs and risk of stroke and stroke mortality. Neurology 2005;65:1005-1009.

2. Liew G, Wang JJ, Cheung N, et al. The retinal vasculature as a fractal: methodology, reliability, and relationship to blood pressure. Ophthalmology 2008;115:1951-1956.

3. Grauslund J, Green A, Kawasaki R, Hodgson L, Sjolie AK, Wong TY. Retinal Vascular Fractals and Microvascular and Macrovascular Complications in Type 1 Diabetes.

Ophthalmology 2010;117:1400-1405.

4. Doubal FN, MacGillivray TJ, Patton N, Dhillon B, Dennis MS, Wardlaw JM. Fractal analysis of retinal vessels suggests that a distinct vasculopathy causes lacunar stroke. Neurology 2010;74:1102-1107.

5. Cheung N, Liew G, Lindley RI, et al. Retinal fractals and acute lacunar stroke. Ann Neurol 2010;68:107-111.

6. Azemin MZ, Kumar DK, Wong TY, et al. Age-related rarefaction in the fractal dimension of retinal vessel. Neurobiol Aging Epub 2010 May 14.

7. Che Azemin MZ, Kumar DK, Wong TY, Kawasaki R, Mitchell P, Wang JJ. Robust Methodology for Fractal Analysis of the Retinal Vasculature. IEEE Trans Med Imaging Epub 2010 Sep 16. 
Table 1. Comparison of characteristics of stroke cases and matched controls.

\begin{tabular}{|c|c|c|c|}
\hline & Controls $(n=184)$ & Stroke cases $(n=101)$ & \\
\hline Matched characteristics & Mean (SD) & Mean (SD) & p-value \\
\hline \multirow[t]{2}{*}{ Age, years } & $73.6(8.1)$ & $74.0(8.5)$ & 0.73 \\
\hline & n (\%) & n (\%) & \\
\hline Gender, male & $78(41.7 \%)$ & $42(41.6 \%)$ & 0.98 \\
\hline Hypertension, present & $89(47.6 \%)$ & $52(51.5 \%)$ & 0.13 \\
\hline Diabetes, present & $7(3.7 \%)$ & $6(5.9 \%)$ & 0.39 \\
\hline Other clinical characteristics & Mean (SD) & Mean (SD) & p-value \\
\hline Systolic blood pressure, $\mathrm{mmHg}$ & $154.5(25.7)$ & $152.7(22.7)$ & 0.55 \\
\hline Diastolic blood pressure, $\mathrm{mmHg}$ & $82.6(10.7)$ & $83.5(10.9)$ & 0.51 \\
\hline Fasting plasma blood glucose,$(\mathrm{mmol} / \mathrm{L})$ & $5.0(1.3)$ & $5.1(4.3)$ & 0.98 \\
\hline$\overline{\text { Body mass index }\left(\mathrm{kg} / \mathrm{m}^{2}\right)}$ & $24.9(3.6)$ & $25.0(4.3)$ & 0.99 \\
\hline Total cholesterol $(\mathrm{mmol} / \mathrm{L})$ & $6.0(1.0)$ & $6.1(1.1)$ & 0.34 \\
\hline HDL choresterol $(\mathrm{mmol} / \mathrm{L})$ & $1.49(0.46)$ & $1.48(0.50)$ & 0.86 \\
\hline \multirow[t]{2}{*}{$\begin{array}{l}\text { Triglycerides }(\mathrm{mmol} / \mathrm{L}) \\
(\text { Median and Inter-quartile range })\end{array}$} & $1.5(1.2-2.1)$ & $1.4(1.1-1.8)$ & 0.08 \\
\hline & n (\%) & n (\%) & \\
\hline Current smoker & $16(8.6 \%)$ & $13(12.9 \%)$ & 0.25 \\
\hline Central Retinal Artery Equivalent $(\mu \mathrm{m})$ & $159.2(1.1)$ & $159.7(1.6)$ & 0.77 \\
\hline \multirow[t]{2}{*}{ Central Retinla Vein Equivalent $(\mu \mathrm{m})$} & $237.7(1.8)$ & $236.1(2.6)$ & 0.62 \\
\hline & Mean $(95 \% \mathrm{CI})$ & Mean $(95 \%$ CI) & p-value \\
\hline SFD of retinal vascular pattern & $1.511(1.507,1.515)$ & $1.504(1.499,1.510)$ & 0.044 \\
\hline
\end{tabular}

HDL: High-density lipoprotein. SFD: Spectrum fractal dimension. Data are expressed as numbers $(\%)$ or means and standard errors. 